distortions, impaired sexual function, decreased quality of life and increased vulvar cancer risk.

An agreement to measure VLS severity in a standard way is yet to be defined and, to our knowledge, no standardized clinical classification of anatomical modifications in VLS has been validated.

The purpose of this study was to prepare a clinical classification for VLS aimed at defining the morphological patterns of this condition, while stratifying them into grades. The classification is intended to provide a homogeneous and reproducible description of the different features of this disease. It

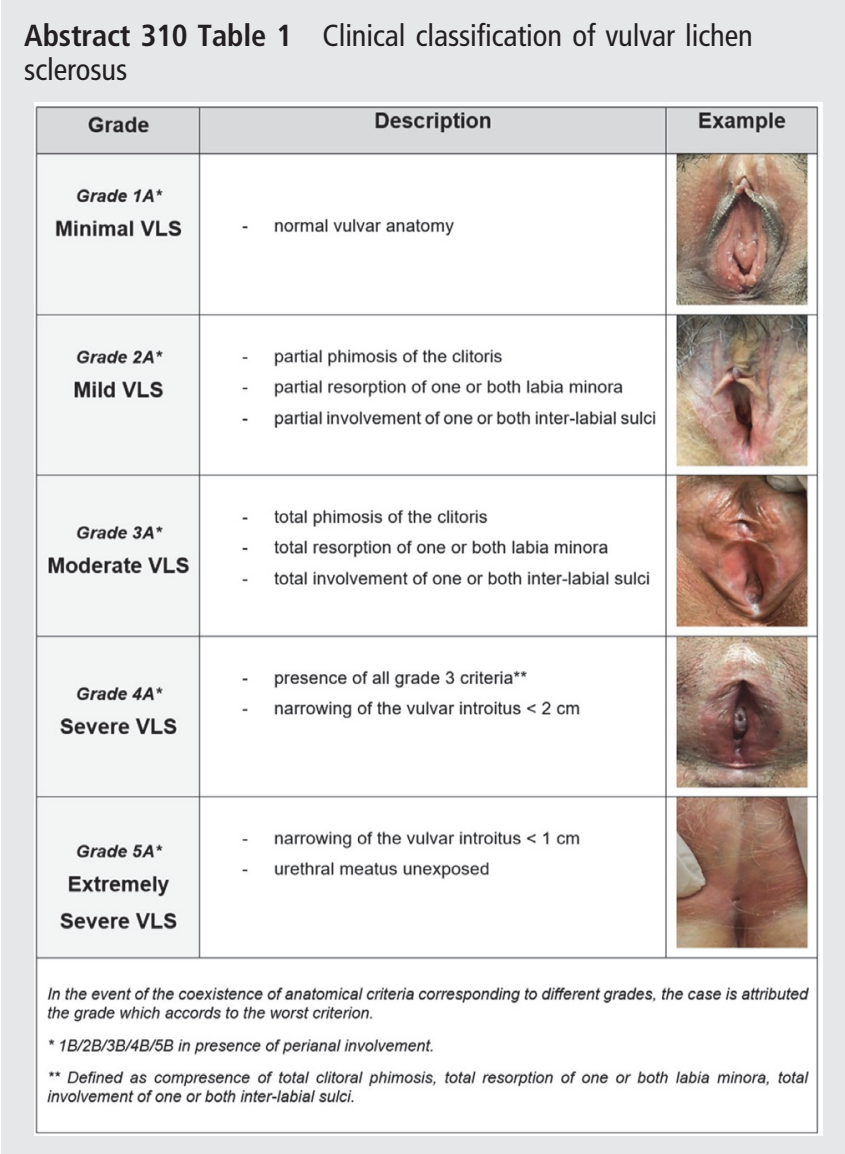

Abstract 310 Table 2 Level of agreement among the raters

\begin{tabular}{|c|c|c|c|c|}
\hline \multicolumn{5}{|c|}{ Agreement among the raters for the 137 cases of VLS } \\
\hline \multicolumn{4}{|c|}{ ICC } & $95 \% \mathrm{Cl}$ \\
\hline \multicolumn{2}{|l|}{ Total panel } & \multicolumn{2}{|c|}{0.89} & $0.87-0.91$ \\
\hline \multicolumn{2}{|c|}{ Board members } & \multicolumn{2}{|c|}{0.92} & $0.89-0.93$ \\
\hline \multicolumn{2}{|c|}{ Gynecologists with no expertise } & \multicolumn{2}{|c|}{0.87} & $0.84-0.90$ \\
\hline \multicolumn{2}{|c|}{ Cases in women aged $<50$ years } & \multicolumn{2}{|c|}{0.92} & $0.86-0.96$ \\
\hline \multicolumn{2}{|c|}{ Cases in women aged $>50$ year } & \multicolumn{2}{|c|}{0.89} & $0.86-0.92$ \\
\hline \multicolumn{5}{|c|}{ Agreement among the raters between the first and the second evaluation. } \\
\hline & Board member 1 & Board member 2 & $\begin{array}{l}\text { Gynecologist no } \\
\text { expertise } 1\end{array}$ & $\begin{array}{c}\text { Gynecologist no } \\
\text { expertise } 2\end{array}$ \\
\hline $\begin{array}{l}\text { Kappa } \\
\text { (95\% CI) }\end{array}$ & $0.91(0.79-1.00)$ & $0.96(0.87-1.00)$ & $0.79(0.60-0.95)$ & $0.91(0.79-1.00)$ \\
\hline $\begin{array}{l}\text { ICC } \\
(95 \% \mathrm{CI})\end{array}$ & $0.97(0.94-0.99)$ & $0.99(0.97-0.99)$ & $0.93(0.86-0.97)$ & $0.97(0.94-0.99)$ \\
\hline
\end{tabular}

also serves as an important tool for the evaluation of the course of the disease over time, response to treatment, and for comparison of clinical studies.

Methodology A board of seven specialists with expertise in vulvar pathology were asked to outline the anatomical criteria for the definition of VLS severity (phimosis of the clitoris, resorption of the labia minora, involvement of the inter-labial sulcus, and narrowing of the vulvar introitus), identifying five grades to be used to build-up of a score model. The classification was validated by 13 physicians upon pictures of 137 consecutive patients. Each physician individually assigned a grade to each case, according to the abovementioned criteria. Interrater agreement among evaluators was analysed by means of ICC (Intraclass Correlation Coefficient). Intra-observer reproducibility and inter-observer concordance in vivo were analysed by means of Kappa index.

Results This study provides a new classification of VLS, based on defined anatomical criteria and graded into mutually exclusive progressive classes (table 1).

The ICC analysis showed a substantial agreement in the attribution of the grade of VLS among the 137 cases, ICC $=0,89$ (0.87-0.91), both in the expert and in the nonexpert group (ICC $=0.92$ and 0.87 respectively). An 'almost perfect' agreement was achieved for intra-observer reproducibility and among physicians in vivo (Kappa 0.93).

Conclusion Our classification showed a high accuracy in defining morphological modifications in VLS. It is easy to use, reproducible, and can be applied by different health care providers in daily clinical practice and in all clinical settings.

Disclosure Nothing to disclose.

\section{DIAGNOSTIC VALUE OF HE4, CA-125, ROMA AND CPH-I FOR PREOPERATIVE ASSESSMENT OF OVARIAN TUMORS}

${ }^{1}$ Núria Carreras Diéguez, ${ }^{1}$ Ariel Glickman, ${ }^{1}$ Núria Agustí, ${ }^{2}$ Marta Del Pino, ${ }^{1}$ Berta DiazFeijoo, 'Jaume Pahisa, 'Pere Fusté, 'Aureli Torne. ${ }^{1}$ Hospital Clínic de Barcelona; Gynecologic Oncology Unit; ${ }^{2}$ Hospital Clínic; Hospital Clinic Barcelona; Gynaecology

\subsection{6/jgc-2020-ESGO.48}

Introduction/Background Among patients with adnexal masses, preoperative identification of epithelial ovarian cancer (EOC) or metastatic cancer in the ovary (MCO) is essential for surgical planning. Our aim was to assess the performance of CA125, HE4, and the probability models, Risk of Ovarian Malignancy Algorithm (ROMA) and Copenhagen Index (CPHI), to preoperatively identify EOC or MCO.

Methodology We performed a single center retrospective study including women who underwent surgery for an ovarian tumor between January 2000 and December 2018. We defined two study groups: one group comprising women with benign pathology and borderline epithelial ovarian tumors and a second group comprising women with EOC or MCO. We computed sensitivity, specificity and predictive values of CA125, HE4, ROMA and CPH-I at different cutoff points. We performed receiver operative curve analysis for tumor markers, CPH-I and ROMA models. We performed subgroup analysis including only premenopausal, postmenopausal women, stage I EOC and women harboring ovarian tumors with inconclusive diagnosis of malignancy by ultrasound features.

Results One thousand seventy-one patients were included, $852(79.55 \%)$ presented benign or borderline epithelial 
Abstract 311 Table 1 Sensitivity, specificity, and predictive values of Ca125, He4, ROMA index and CPH-1 index at different cutoff points

\begin{tabular}{|c|c|c|c|c|}
\hline Parameter (cutoff) & Sensitivity $(95 \% \mathrm{CI})$ & Specificity $(95 \%$ CI) & PPV $(95 \% \mathrm{CI})$ & $\mathrm{NPV}(95 \% \mathrm{CI})$ \\
\hline Ca125 $(100 \mathrm{U} / \mathrm{mL})$ & $61.86(55.21-68.09)$ & $92.71(90.73-94.29)$ & $68.91(62.07-75.02)$ & $90.30(88.11-92.11)$ \\
\hline Ca125 (35/65 U/mL)* & $74.42(68.23-79.81)$ & $80.70(77.81-83.24)$ & $50.21(44.73-55.64)$ & $92.30(90.22-94.12)$ \\
\hline $\operatorname{Ca} 125(35 / 100 \mathrm{U} / \mathrm{mL})^{*}$ & $66.98(60.44-72.92)$ & $81.39(78.58-83.90)$ & $48.48(42.86-54.15)$ & $90.41(88.07-92.32)$ \\
\hline $\mathrm{He} 4(70 \mathrm{pmol} / \mathrm{L})$ & $83.25(77.31-87.88)$ & $86.11(83.40-88.43)$ & $61.15(55.11-66.87)$ & $95.14(93.22-96.53)$ \\
\hline $\mathrm{He} 4(120 \mathrm{pmol} / \mathrm{L})$ & $69.11(62.23-75.23)$ & $96.29(94.65$ - 97.44) & $83.02(76.42-88.06)$ & $92.23(90.10$ - 93.93) \\
\hline $\mathrm{He} 4(70 / 140 \mathrm{pmol} / \mathrm{L})^{*}$ & $70.70(63.93-76.71)$ & $93.5(91.52-95.11)$ & $74.2(67.40-80.01)$ & $92.4(90.20-94.10)$ \\
\hline ROMA (10) & $94.24(89.98-96.75)$ & $72.71(69.27-75.91)$ & $47.91(43.84-54.01)$ & $97.85(96.19-98.80)$ \\
\hline ROMA (15) & $91.10(86.21-94.37)$ & $84.62(81.73-87.12)$ & $62.14(56.33-67.62))$ & $97.17(95.51-98.22)$ \\
\hline ROMA $(12.5 / 14.4)^{*}$ & $91.62(86.83-94.78)$ & $82.58(79.57-85.23)$ & $59.32(53.63-64.77)$ & $97.26(95.60-98.31)$ \\
\hline ROMA (13.1/27.7)* & $84.8(79.00-89.20)$ & $89.0(86.40-91.10)$ & $68.1(61.90-73.70)$ & $95.5(93.60-96.80)$ \\
\hline $\mathrm{CPH}(\mathrm{I})$ & $96.86(93.32-98.55)$ & $47.97(44.26-51.70)$ & $34.01(30.15-38.09)$ & $98.22(96.17-99.18)$ \\
\hline $\mathrm{CPH}(3)$ & $91.10(86.22-94.42)$ & $79.12(75.91-82.01)$ & $54.72(49.23-60.14)$ & $97.01(95.21-98.12)$ \\
\hline CPH (5) & $86.91(81.39-90.97)$ & $87.54(84.86-89.79)$ & $65.87(59.82-71.45)$ & $96.03(94.20-97.29)$ \\
\hline $\mathrm{CPH}(7)$ & $82.20(76.16-86.97)$ & $90.87(88.49-92.80)$ & $71.36(65.06-76.93)$ & $94.86(92.90-96.309)$ \\
\hline
\end{tabular}

PPV: positive predictive value; $N P V:$ negative predictive value;

*premenopausal/postmenopausal women

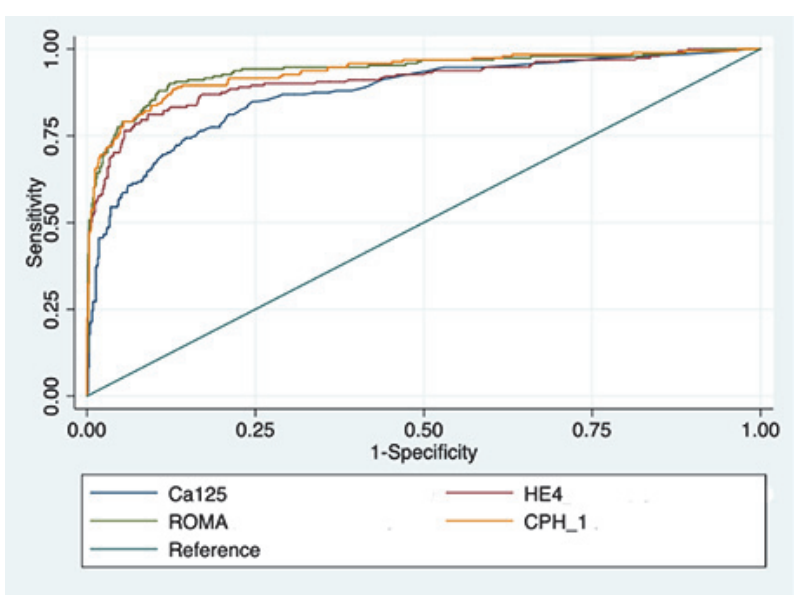

Abstract 311 Figure 1 AUC of He4, Ca125, ROMA and CPH-I. All patients

tumors and $219(20.45 \%)$ presented EOC or MCO. Area under the curve (AUC) for HE4 was significantly higher than AUC for CA125 (0.909 vs 0.873). No differences were seen between AUC of ROMA (0.939) and CPH-I (0.936), but they were both higher than HE4 AUC (figure 1). Subgroup analysis showed that in premenopausal women, HE4 performed better than CA125, and was equivalent to ROMA or $\mathrm{CPH}-\mathrm{I}$. Considering only ovarian tumors with inconclusive diagnosis by ultrasound, ROMA performed better than $\mathrm{CPH}-$ I. None of the tumor markers alone achieved a sensitivity of 90\%, however HE4 was highly specific (93.5\%). ROMA showed a sensitivity and specificity of $91.1 \%$ and $84.6 \%$ respectively, while CPH-I showed a sensitivity of $91.1 \%$ with 79.2\% specificity (table 1 ).

Conclusion Both ROMA and CPH-I were more favorable than tumor markers alone for differentiating patients harboring EOC or MCO. Probability models can be helpful in order to assess the risk of malignancy of ovarian tumors, especially when expert ultrasound examination is not available or when the diagnosis by ultrasound remains inconclusive.

Disclosures The authors of this abstract have no disclosures.

\section{ONE-STEP NUCLEIC ACID AMPLIFICATION (OSNA): A BIG 'STEP' TOWARDS A MORE ACCURATE INTRA- OPERATIVE ASSESSMENT OF SENTINEL LYMPH NODE STATUS FOR EARLY STAGE ENDOMETRIAL AND CERVICAL CANCER?}

${ }^{1}$ Anastasios Tranoulis, ${ }^{2}$ Dimitra Georgiou, ${ }^{3}$ Stephen Attard Montalto, ${ }^{1} J a s o n$ Yap, ${ }^{4}$ Jeremy Twigg, ${ }^{1}$ Felicia Buruiana, ${ }^{1}$ Sudha Sundar, ${ }^{1} J a n o s$ Balega, ${ }^{1}$ Kavita Singh, ${ }^{1}$ Sean Kehoe. ${ }^{1}$ The Pan-Birmingham Gynaecological Oncology Centre, Sandwell and West Birmingham NHS Trust, Birmingham; '2Department of Gynaecological Oncology, Chelsea and Westminster NHS Trust, Imperial College, London; ${ }^{3}$ Department of Gynaecological Oncology, Maidstone and Tunbridge Wells NHS Trust, Maidstone; ${ }^{4}$ Department of Gynaecological Oncology, University Hospitals Coventry and Warwickshire NHS Trust, Coventry

\subsection{6/ijgc-2020-ESG0.49}

Introduction/Background Sentinel lymph node (SLN) mapping is established as the standard of care for staging in selected cases of melanoma, breast and vulval cancer amongst other malignancies. Nonetheless, adapting its use in endometrial cancer (EC) and cervical cancer (CC) has been challenging. There is currently growing evidence to support its accuracy in earlystage EC and CC. One-step nucleic acid amplification (OSNA) has emerged as a rapid molecular assay for the detection of cytokeratin 19-mRNA in SLNs. The aim of the study was to ascertain the accuracy of OSNA in detecting SLN metastasis in early-stage EC and CC compared to that of histopathological ultra-staging.

Methodology A systematic search of MEDLINE, SCOPUS, ClinicalTrials.gov, and Cochrane Database was performed, spanning the period Jan 1975 to July 2020. Studies pertaining to the use of OSNA in detecting SLN metastasis in EC and CC were located. Pathologic ultra-staging was the reference standard. The quality of the included studies was assessed using the QUANDAS-2 tool. The DerSimonian-Laird randomeffects model was used. Sensitivity, specificity, positive and negative likelihood ratio (LR+/LR-), diagnostic odds ratio (DOR), and the area under the curve (AUC) on SROC curve were calculated. We assessed the inter-study heterogeneity by the Higgin's I2 index. Statistical analysis was performed using the STATA version 16.1. 